\title{
Spontaneous and Induced Star Formation in the LMC
}

\author{
Yu.N. Efremov \\ Sternberg Astronomical Institute, MSU, Moscow 199899, Russia \\ B.G. Elmegreen \\ IBM Watson Researcher Center, Yorktown Heights, NY 10598, USA
}

\begin{abstract}
Spontaneous and triggered star formation in the LMC is discussed with data on star clusters ages and positions. The supershell LMC4 and the stellar arcs in the same region are suggested to be triggered by GRBs, the progenitors of which might have escaped the old elliptical cluster NGC1978, close to which are a number of X-ray binaries and the SGR/SNR N49.
\end{abstract}

\section{Stochastic Star Formation}

The LMC is the best site to study large-scale features of star formation as displayed by mutual positions and ages of star clusters. The ages are now available for about 600 clusters.

Efremov \& Elmegreen (1998a) demonstrated recently that the larger the mutual distance between these clusters, the larger their age difference. This relation may be considered part of a general correlation between the size of a star formation region and the duration of star formation there. For example, OB subgroups each form in $\sim 3 \mathrm{My}$, the whole OB associations that surround them form in $\sim 10 \mathrm{My}$, and the star complexes, such as Gould's Belt, that include the $\mathrm{OB}$ associations, form in $\sim 30 \mathrm{My}$; each scale is larger than the previous by a factor of 10 (e.g., $3 \mathrm{pc}, 30 \mathrm{pc}$, and $300 \mathrm{pc}$ ). Thus the duration of star formation scales approximately with the square root of the size of the region in this example (see review in Elmegreen \& Efremov 1999).

Such scaling is expected for star formation in turbulent gas, because star formation usually operates on a time scale proportional to the cloud or clump crossing time, and this crossing time scales with the square root of region size. Thus the formation of hierarchical young stellar groups results from hierarchical cloud structure. There is no preferred scale for cluster formation.

\section{Triggered Stellar Arcs in the LMC4 Region}

The LMC harbours what is often considered to be the classical region of triggered star formation, the LMC4 supershell identified and photographed in $\mathrm{H} \alpha$ by Meaburn (1980). This region is sometimes erroneously called Shapley's Constellation III, but that title should go to the region around the NGC 1974 cluster 
(see Efremov \& Elmegreen 1998b). Three or four gigantic arcs of stars and clusters are known in the LMC4 region (Hodge 1967). The most obvious arc was considered by Westerlund \& Mathewson (1966): it is inside the southern part of the supergiant HI hole/shell, found by McGee \& Milton (1966), Domgörgen et al. (1995), and Kim et al. (1997). Westerlund \& Mathewson (1966) ascribed the origin of the stellar arc and HI features to the outburst of a Super-Supernova, following Shklovsky (1960). Later on, the region was considered the best manifestation of triggered self-propagated star formation (Dopita et al. 1985). However, there are serious difficulties with this interpretation if the brightest stars in the center of the $\mathrm{HI}$ ring are considered to be the triggering cluster, because these stars are too young (Olsen et al. 1997; Braun et al. 1997).

Recently, Efremov \& Elmegreen (1998b) suggested that two well-shaped arcs in this region formed by triggered star formation in gas that was sweptup by centralized sources of pressure. The strictly circular shapes of both arcs are the strongest evidence for this. Six coeval A I stars near the center of the larger arc (called Quadrant) were suggested to be the remnants of an association, including O-stars, which swepted up the gas in the larger region starting $\sim 30$ My ago. A small and younger cluster near the center of the smaller arc (Sextant) was shown to be responsible for that one.

These centralized stellar sources of pressure could produce both young stellar arcs at the right time and position, as Efremov \& Elmegreen (1998b) demonstrated, yet the general picture is still not satisfactory. The main questions remaining are:

1. Why are there no giant stellar arcs or rings around other, even more rich, clusters in the LMC?

2. Why are all of the stellar arcs in the LMC close to each other in only this place?

3. Why are there just arcs and not full stellar rings?

4. Why are the arcs in the region of the largest and deepest $\mathrm{HI}$ hole in the LMC?

The recent identification of Gamma-ray burst (GRB) afterglows in distant galaxies revived the possibility that single super-explosions can produce large shells and trigger star formation (Efremov, Elmegreen \& Hodge 1998; Perna \& Loeb 1998). There may not even be a central cluster or evidence of an extragalactic cloud impact in the triggered region. Indeed, the arcs in the LMC4 region, and the whole HI supershell as well, could be produced by GRB-like explosions (Efremov, Elmegreen \& Hodge, 1998).

This explains question (1) above, yet not the other three. Why would all the peculiar outbursts occur in the same region of the LMC? An answer to this question might come if we consider the common assumption that explosions of some GRBs are the result of merging components of close binaries that include a neutron star, black hole or white dwarf. This hypothesis was recently advanced by Efremov (1998) to account for the stellar arcs and LMC4 supershell; the binaries could have escaped from NGC 1978, which is a massive cluster of intermediate age in the same area of the LMC, up and right of $2^{\prime \prime}$ within the circle in Figure 1 by Efremov \& Elmegreen (1998b). 


\section{NGC1978 as the Origin of GRB Progenitors}

The high rate of occurrence of X-ray binaries (with one component a neutron star) inside dense globular clusters is well known (e.g., Baylin 1996). It was explained long ago as consequence of the high probability of formation of close binaries after tidal captures in the dense cluster (e.g., Shklovsky 1982; Davies 1995). It was also shown (McMillan 1986) that a large number of tidally captured binaries may escape a dense old cluster as the result of three-body collisions. Recently Hanson \& Murali (1998) suggested that stellar encounters in globular clusters were able to produce not only millisecond pulsars, but also binaries that evolve into GRBs.

The formation of a neutron star after a SN explosion in a binary system leads to a high recoil velocity, the most likely value of which is $150-200 \mathrm{~km}^{-1}$ (Lipunov et al. 1997). Such high velocities would spread out any future GRB over very large distances around the paternal cluster. Even smaller velocities would disperse the GRB progenitors significantly, because the binaries may take $100 \mathrm{My}$ before they merge to give a GRB (Lipunov et al. 1997). We therefore suggest that the relics of GRB might be observed in the kiloparsec-scale regions surrounding old dense clusters.

NGC 1978 is a rich and old cluster, though much younger than classical globulars in the Milky Way and the LMC. Its age is about $2 \times 10^{9}$ (Bomans et al. 1995), and it is the richest such cluster in the LMC. Indeed, it has a few hundred red giants with masses of around $1 \mathrm{M}_{\odot}$ !

NGC 1978 is also unusual in its extremely flattened shape (Geisler \& Hodge 1980). This may indicate a formation process involving the merger of two clusters, especially because no rotation has been detected (Fisher et al. 1992). Besides the shape, there is no other evidence for merging, yet Kravtsov (1999) found some signs of abundance differences in two parts of the cluster. The process of merging surely increases the probability of stellar encounters, leading both to the formation of close binaries and to the escape of many stars from the resulting cluster (e.g., de Oliveira et al. 1999). The observation of a large number of blue stragglers in NGC 1978 (Cole et al. 1997) might also indicate a high rate of stellar encounters there.

There are also other objects near NGC 1978 that are binary stars with a compact component and are therefore related to GRB progenitors if they are not progenitors themselves. Three X-ray binaries are within $20^{\prime}$ of NGC 1978 and more are in a wider surrounding, as is evident from Haberl et al. (1999). These objects are ascribed mostly to high-mass binaries, yet some classified as $\mathrm{Be} / \mathrm{X}$-ray binaries might contain white dwarfs (see Neguerula 1998).

Moreover, the famous GRB of March 5, 1979 is also at about $20^{\prime}$ to the NW of the cluster. It is now known as the Soft Gamma Ray (SGR) repeater SGR 0526-66, and it is an X-ray binary, i.e., a stellar remnant of a SN inside the young remnant N49 (Danner et al. 1998).

This is consistent with the conclusion of Nakamura (1998) that low energy GRBs leave behind SGR repeaters. Nakamura explained in this way the properties of SN1998bw (of b/c type), which was a bright Supernova and also a GRB with low energy (Woosley et al. 1999). All three well-studied SGRs are indeed 
connected with young SNRs (Kouvelitou et al. 1998). The connection between SGRs and GRBs was recently suggested by Wang \& Wheeler (1998).

We propose that the outburst that produced SGR 0526-66 plus SNR N49 was similar to the SN1998bw/GRB980425 event, and also that events like these produced the stellar arcs near LMC4. Indeed, the Quadrant and Sextant arcs might be produced by the stellar winds and supernovae from one to five dozen O-type stars (Efremov \& Elmegreen 1998b), which corresponds approximately to 10 to 50 common SNs. The energy from this is comparable to or a bit below that of common GRBs. An energy 10-100 times higher was necessary to form the entire LMC4 $\mathrm{HI}$ hole; this is within the energy range observed for GRBs. We noted that a second, rich, intermediate-age cluster in the LMC, NGC1806, is inside the HII supershell LMC6, in the region of low HI density.

\section{Conclusions}

Stellar arcs near LMC4, and the LMC4 HI hole itself, might have been produced by GRBs whose progenitors originated in the intermediate-age, nearby, globular cluster NGC 1978. GRBs like this may occur anywhere in a galaxy near such a dense cluster, whereas sequential SNs in young clusters might only occur in the spiral arms and near the planes of galactic disks. Indeed, the whole LMC4 region and other similar patches of star formation with giant stellar arcs (as in M83 and NGC6946 - see Efremov, Elmegreen \& Hodge 1998) might be produced by GRBs. The arcs might be only parts of circles (e.g., Hodge 1967; Efremov 1998) because the GRBs explode outside the galactic plane. The abundance of SNs in both M83 and NGC 6946 might be one more indication of a connection between certain SNs and GRBs.

One important issue that is still unresolved is why other dense globular clusters in the LMC and elsewhere have no similar concentrations of GRB relicts. For example, Ciardullo et al. (1990) suggested that the large number of $\mathrm{X}$-ray binaries in the bulge of M31 might be the result of ejection from the globular clusters there. The unique properties of NGC 1978 that might have led to the observed stellar arcs are its elliptical shape (suggesting a merger) and its high abundance of red giants (which are more massive stars than in classical globulars). These properties are plausibly connected with the occurrence of surrounding GRB relics and X-ray binaries. Perhaps the compact binaries ejected from this cluster evolve not only to X-rays sources, but to SN/SGR and GRBs as well.

\section{References}

Baylin, C.D. 1996, ASP Conf. Ser., 90, 320

Bomans, D.J., Vallenari, A., \& de Boer, K.S. 1995, A\&A, 298, 427

Braun, J.M., Bomans, D.J., Will, J.-M., \& de Boer, K.S. 1997, A\&A, 328, 167

Ciardullo, R., Tamblyn, P., \& Phillips, A.C. 1990, PASP, 102, 1113

Cole, A.A., et al. 1997, AJ, 114, 1945

Danner, R., Kulkarni, S.R., \& Trumper, J. 1998, BAAS, 192, No. 43.09 
de Oliveira, M., Dottori, H., \& Bica, E. 1999, this volume

Domgörgen, H., Bomans, D.J., \& de Boer, K.S. 1995, A\&A, 296, 523

Davies, M. 1995, MNRAS, 276, 887

Dopita, M.A., Mathewson, D.S., \& Ford, V.L. 1985, ApJ, 297, 599

Efremov, Yu.N. 1999, Pis'ma Astron. Zh., in press

Efremov, Yu.N., \& Elmegreen, B.G. 1998a, MNRAS, 299, 588

Efremov, Yu.N., \& Elmegreen, B.G. 1998b, MNRAS, 299, 643

Efremov, Yu.N., Elmegreen, B.G., \& Hodge P.W. 1998, ApJ, 501, L163

Elmegreen, B.G., \& Efremov, Yu.N. 1999, in The Orion Complex Revisited, ed. M. J. McCaughrean \& A. Burkert, ASP Conference Series, 1999, in press.

Geisler, D., \& Hodge, P. 1980, ApJ, 242, 66

Haberl, F., Pietsch, W., \& Filipovic, M. 1999, this volume

Meaburn, J. 1980, MNRAS, 192, 365

Hanson, B.M.S., \& Murali, C. 1998, ApJ, 505, L15

Hodge, P.W. 1967, PASP, 79, 29

Kim, S., Staveley-Smith, L., Sault, R.J., Kesteven, M.J., McConnell, D., \& Freeman, K.C. 1997, PASA, 14, 119

Kravtsov, V. 1999, this volume

Kouvelitou, C. et al. 1998, Nature, 393, 235

Lipunov, V.M., Postnov, K.A., \& Prokhorov, M.E. 1997, MNRAS, 288, 245

Loeb, A., \& Perna, R. 1998, ApJ, 503, L35

MacMillan, S.L. 1986, ApJ, 307, 126

McGee, R.X., \& Milton, J.A. 1966, Aust. J. Phys., 19, 343

Nakamura, T. 1998, submitted to Prog. Theor. Phys. (astro-ph/9807087)

Negueruela, I. 1998, A\&A, 338, 505

Olsen, K.A.G., Hodge, P.W., Wilcots, E.M., \& Pastwick, L. 1997, ApJ, 475, 545

Shklovsky, I.S. 1960, Soviet Astron. - AJ, 4, 355

Shklovsky, I.S. 1982, Comments on Astrophys., 9, 261

Wang, L., \& Wheeler, J.C. 1998, ApJ, 504, L87

Westerlund, B.E., \& Mathewson, D.S. 1966, MNRAS, 131, 371

Woosley, S.E., Eastman, R.G., \& Schmidt, B.P. 1999, ApJ, 516, 788

\section{Discussion}

Lance Gardiner: There is evidence of asymmetrical spiral structure in the LMC from the analysis of the spatial distribution of young star clusters by Doffer et al. Numerical simulations have shown that an asymmetric spiral structure can be induced by an off center bar, as in the LMC. Is the hierarchical distribution of young stars you found consistent with the existence of spiral structure in the LMC?

Efremov: The star-forming spiral arms are just the highest level in the hierarchy of star-forming regions, so if they exist in the LMC, our conclusions on the 
hierarchical star formation are not affected. The density wave arms do surely not exist in the LMC, and even the sheared spiral fragments are seen probably only in the outer HI disk, as Kim's data revealed.

Nolan Walborn: Do you think a configuration of alignments such as you propose would be maintained for $10^{8}$ years?

Efremov: The supergiant HI shells may live up to $100 \mathrm{Myrs}$ or so if there is no shear and the HI disk thick enough. This is why these supershells are found usually in irregular galaxies and in the outer parts of spiral galaxies. As concerns stellar arcs, the dynamical evaluations of their lifetime could be of great use. Surely the arcs cannot live long, the Quadrant arc is already more dispersed than the Sextant arc (ages are $\approx 15$ and $\approx 7$ Myrs respectively). 


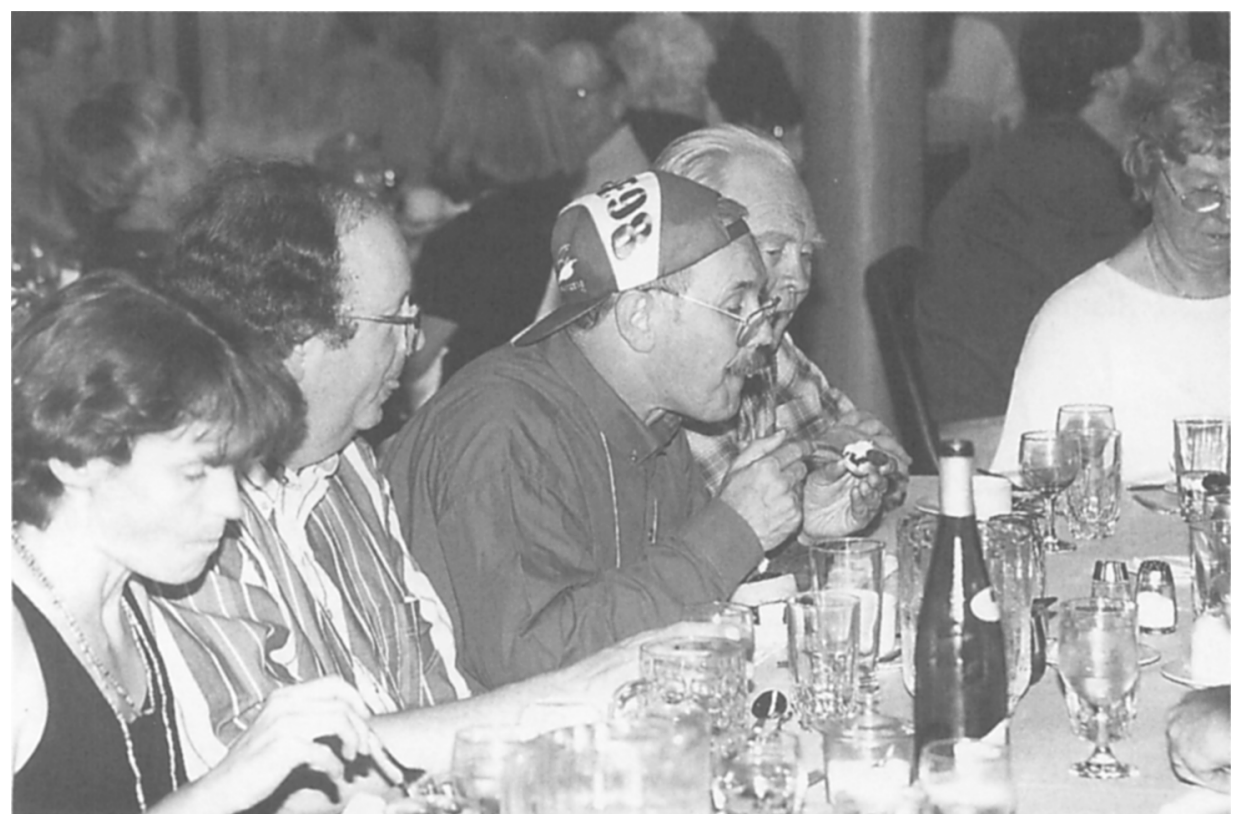

During the banquet, Rebecca Elson, Jay Gallagher, and Bengt and Vivi Westerlund contemplate whether Hans Zinnecker wears his World Cup cap while sleeping, too. 